\title{
Repair Preparation of Fiber-Reinforced Plastics by the Machining of a Stepped Peripheral Zone
}

\author{
David Zaremba ${ }^{1,}{ }^{*}$ - Christian Biskup ${ }^{1}-$ Thomas Heber $^{2}$ - Nico Weckend ${ }^{2}-$ \\ Werner Hufenbach ${ }^{2}$ - Frank Adam ${ }^{2}$ - Friedrich-Wilhelm Bach ${ }^{1}$ - Thomas Hasse ${ }^{1}$ \\ ${ }^{1}$ Leibniz Universität Hannover, Germany \\ 2 Technische Universität Dresden, Germany
}

\begin{abstract}
Today's standard procedures for the repair of fiber reinforced plastics are not optimized for the structural rearrangement of the original material properties. Alongside lap repair and scarf repair, a newly introduced method for the machining of a stepped peripheral zone is discussed. For this purpose, the methods of dry ice blasting and snow blasting as well as the water jet are being investigated. Reference material is carbon fiber reinforced plastic (CFRP) compliant to laminates used in the aviation industry.

It was found that snow blasting and dry ice blasting were not suitable for this purpose in the experimental set-up. In contrast, the water jet allowed a precise control of the material removal. Subsequently to the feasibility study, a parameter study was carried out to determine applicable parameters for the surface preparation of CFRP. After successful machining of a stepped peripheral zone, a repair experiment was carried out with promising results.
\end{abstract}

Keywords: surface preparation, carbon fiber reinforced plastic, CFRP, water jet, dry ice blasting, snow blasting, CFRP repair, stepped peripheral zone

\section{O INTRODUCTION}

Initiated by the lightweight construction trends during the last few years, a certain group of composite materials has gained an increased interest in the industry: fiber-reinforced plastics (FRP) with a thermo- or duroplastic matrix. Materials out of this group find extensive use in aeronautical and aerospace engineering, as well as in vehicle construction and many other application areas, where high strength and low weight are required.

Up to now, a big disadvantage of fiber-reinforced plastics has been their reparability in case of damage [1]. This is primarily based on the fact that the individually designed mechanical properties of such a material are dependent of shape and orientation of the fiber material, which have to be rebuilt.

For the repair of carbon fiber reinforced plastics (CFRP) different mechanisms have been discussed. These mechanisms include different forms of lap repair, where additional repair plies are attached to the surrounding surfaces [2]. Ahn et al. presented different designs methods for this repair technique (stepped lap, uniform lap, single/double-sided repair). Certainly, the basic structural properties of fiber reinforced plastics are not reconstructed using these methods. Instead, loads are led to bypass the damaged or empty section through the additional material.

Unlike lap repair, scarf repair methods are intended to restore the original load distribution and are therefore the repair method of choice. Repairs according to this approach have been made with soft patches [3], as well as with hardened patch material
[4]. Ahn et al. found that the use of prepreg patches results in higher failure loads than the use of wet layups at room temperature. For the additional support of scarf repairs, external repair plies can be applied to seal the repair area.

As introduced by Ahn and Baker, the applied methods are nowadays mostly based on manual machining of the damaged parts. In addition, Baker investigated the adoption of moulded and $\mathrm{CNC}$ machined hard-patches using scarf repair in order to avoid material inhomogenities caused by manual handling. Unfortunately, this method requires a high machining effort and is not practicable. Furthermore, no mechanical values of repaired material have been published.

Using scarf repair, even for small defects a large bonding area is required in order to minimize the stiffness difference between the damaged material and the patch [5]. Beyond that, the risk of material property variations is given because of a high manual machining effort. Consequently, there is a need for automated processing procedures.

However, a reproducible preparation of the composite material is necessary to investigate the aspect of quality in the repair process sufficiently.

The jet machining of 3-dimensional free-form surfaces has successfully been researched by Öjmertz [6] and Borkowski [7]. Due to the use of ceramics and aluminium as the workpiece, the material removal was managed by the abrasive water jet (WAIS) in both publications. When machining FRP, this method would result in an immediate cut-through of the workpiece. Therefore, less abrasive methods are 
researched. Pilot testing has shown that a selective removal of individual CFRP and GFRP layers is possible in general. This gives the new opportunity to adjust the geometric shape of the peripheral repair zone to the patch material. By removing single material layers in a stepped form, a defined surface for the integration of the pre-impregnated (prepreg) patch material is created.

\section{MATERIALS AND METHODS}

Several jetting methods with different materialremoval mechanisms offer a high potential for the selective removal of individual FRP layers. Promising methods include snow blasting, dry ice blasting and pure water jetting. The qualification of these techniques for the manufacturing of a stepped peripheral zone (Fig. 1) will be analyzed and discussed in this paper.

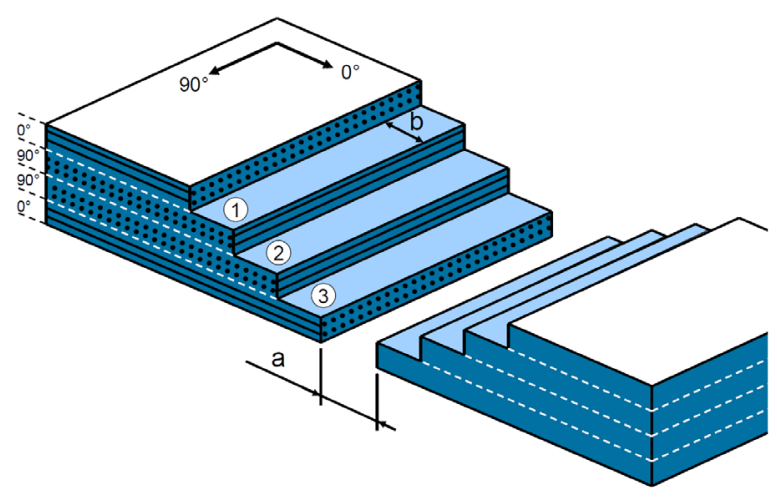

Fig. 1. Stepped peripheral zone for optimal patch integration (step width $b$ and gap size a)

\subsection{Dry Ice Blasting}

Dry ice blasting can be separated into two groups depending on how the dry ice supply is provided. The usage of dry ice blocs requires a breakup into particles immediately before the blasting process. The variety of particle sizes in this group is comparatively high. In order to provide a preferably homogenous process, the application of ready-to-use dry-ice pellets is the more suitable option [8].

The pellets are fed into an air blast by a vibrating plate with an attached metering unit. The resulting mixture of pressurized air and dry ice pellets is led to the nozzle through a flexible tube. Subsequently, the blast is led to the work piece, where the dry ice acts as the blasting agent.

\subsection{Snow Blasting}

Similar to the dry ice blasting, $\mathrm{CO}_{2}$ snow blasting can be classified as a compressed-air blasting method. Liquid carbon dioxide is led to a chamber, where it is subsequently expanded through a restrictor. At this point, the Joule-Thomson effect causes an abrupt cooling down to temperatures lower than $-78.5^{\circ} \mathrm{C}$. By this effect, a part of the carbon dioxide freezes to solid snow particles. The particles are gripped by the air blast and accelerated towards the work piece through a convergent-divergent nozzle.

\subsection{Water Jet}

In the last decades, the high pressure water jet technology has expanded to an important industrial branch. The main applications are the cleaning and cutting (abrasive water jet as well as pure water jet) of a wide-spread material variety [9], other common methods are e.g. peening and surface preparation [10]. Basically, the water jet is generated by decompression from the operating pressure in a nozzle. It can either be used for an erosive impact directly on the work piece, or, in case of the abrasive water injection jet (AWIJ), to accelerate an abrading medium [11]. For the designated purpose, the pure water jet is used.

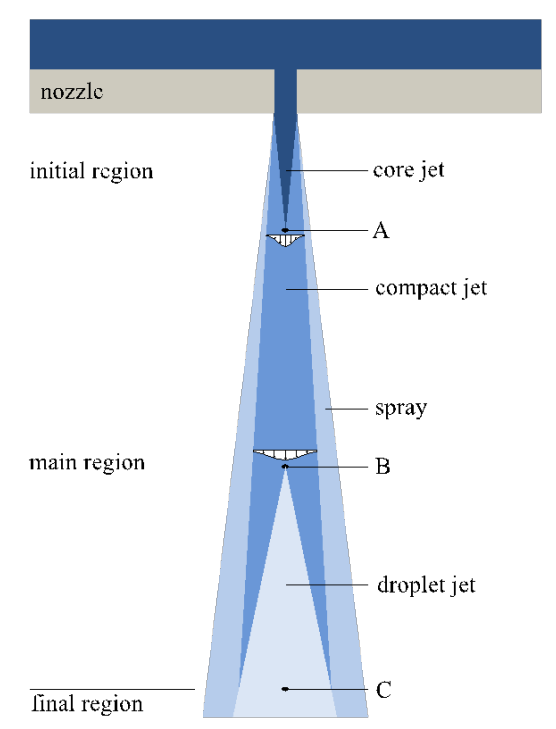

Fig. 2. Schematic states of a water jet, according to [8] and [9]

Caused by environmental effects as well as inner turbulences, the water jet alters its geometrical form and its fluid dynamical characteristics in dependence of its distance to the water nozzle. The basic assumption is that the forming water jet has a constant 
velocity profile directly in the nozzle section. Induced by friction with the environment, behind the nozzle a mixing zone develops subsequently and grows larger with increased distance to the nozzle [9]. As a result, the jet expands (compact jet, see Fig. 2).

For larger nozzle distances, the water jet changes its characteristics again and forms droplets [9]. The impact of single droplets can significantly generate higher peak loads than a continuous jet, because every impact causes stress to the work piece for a short time [10].

\subsection{Experimental Setup}

The test material for all experiments was a symmetrical $\left(0^{\circ} / 90^{\circ} / 90^{\circ} / 0^{\circ}\right)$ CFRP layup (Fig. 3). Each prepreg layer had a thickness of $0.3 \mathrm{~mm}$. Corresponding to common methods in the aviation industry, the laminates were hardened using an autoclave.

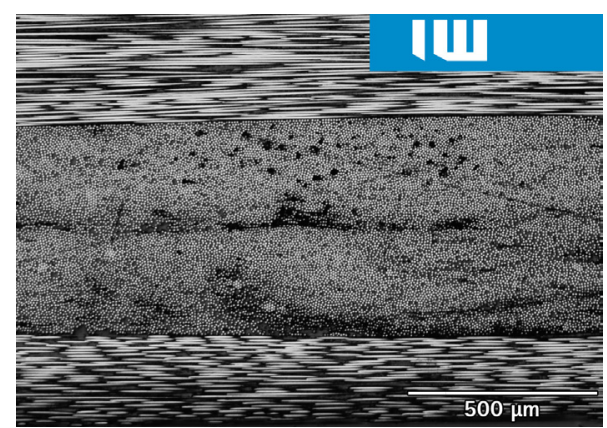

Fig. 3. Microsection of an intact $\left(90^{\circ} / 0^{\circ} / 0^{\circ} / 90^{\circ}\right)$ CFRP layup

The dry ice blasting unit used was a "Linde Cryoclean Cryomax Plus", attached to a 3-axis industrial robot. Used parameters are listed in Table 1. The feed rate of $0 \mathrm{~mm} / \mathrm{s}$ is equivalent to a stationary jet. All experiments were carried out with a $27 \times 5 \mathrm{~mm}$ flat nozzle, both with and without masking. The tool was guided orthogonally to the work piece.

Table 1. Parameters for dry ice blasting

\begin{tabular}{lcc}
\hline \multicolumn{1}{c}{ Parameter } & min. & max. \\
\hline Air blast pressure $[\mathrm{MPa}]$ & 0.2 & 1.5 \\
\hline Shaker pressure $[\mathrm{MPa}]$ & 0.1 & 0.15 \\
\hline Feed rate orientation $\left[^{\circ}\right]$ & 0 & 90 \\
\hline Working distance $[\mathrm{mm}]$ & 30 & 100 \\
\hline Feed rate $[\mathrm{mm} / \mathrm{s}]$ & 0 & 160 \\
\hline Cycles & 1 & 100 \\
\hline
\end{tabular}

For the $\mathrm{CO}_{2}$ snow blasting a "CryoSnow SJ-10" unit with an attached "CryoSnow JP-10" blasting pistol was used. It was attached to the same guiding machine as in the dry ice blasting application.
Experiments were made with three different blasting nozzles. These included two round nozzles (diameter 6 and $11 \mathrm{~mm})$ and one flat jet nozzle $(12 \times 3 \mathrm{~mm})$. The constant $\mathrm{CO}_{2}$ bottle pressure of $5.7 \mathrm{MPa}$ at a temperature of $20{ }^{\circ} \mathrm{C}$ allowed the determination of the fill level by weight measurement. The flow was controlled by an integrated flow regulator. A pressurereducing valve made the regulation of the air blast pressure possible. Used parameters are listed in Table 2.

Table 2. Parameters for snow blasting

\begin{tabular}{lcc}
\hline \multicolumn{1}{c}{ Parameter } & min. & max. \\
\hline Air blast pressure $[\mathrm{MPa}]$ & 0.2 & 1.5 \\
\hline C02 mass flow rate $[\mathrm{g} / \mathrm{s}]$ & 1.67 & 5 \\
\hline Feed rate orientation $\left[{ }^{\circ}\right]$ & 0 & 90 \\
\hline Working distance $[\mathrm{mm}]$ & 30 & 100 \\
\hline Feed rate $[\mathrm{mm} / \mathrm{s}]$ & 0 & 15 \\
\hline
\end{tabular}

For the feasibility study, the blasting pistol was oriented vertically to the work piece. The axis manipulation was started, when erosion on the work piece was evident.

For the water jet experiments, a "Baldor" CNC guiding machine was used with a "Uhde HP19/37" high pressure intensifier pump. Used nozzles were standard sapphire nozzles with diameters from 0.1 $\mathrm{mm}$ up to $0.25 \mathrm{~mm}$. The varied parameters are listed below (Table 3).

Table 3. Water jet parameters

\begin{tabular}{lcc}
\hline \multicolumn{1}{c}{ Parameter } & min. & max. \\
\hline Jet pressure [MPa] & 30 & 400 \\
\hline Feed rate orientation $\left[^{\circ}\right]$ & 0 & 90 \\
\hline Working distance $[\mathrm{mm}]$ & 45 & 100 \\
\hline Feed rate $[\mathrm{mm} / \mathrm{s}]$ & 0 & 15 \\
\hline Nozzle diameter $[\mathrm{mm}]$ & 0.1 & 0.25 \\
\hline
\end{tabular}

The material removal of all specimens was measured using a "Rodenstock RM-600" laser measuring device. All experiments were carried out in two feed rate directions, depending on the orientation of the first fiber layer. In the first instance, the design of experiments scheduled a feasibility study for all three blasting/jetting methods. In the next step, an ensuing parameter test was done for the method with the best test results.

The repaired laminates were prepared for tension testing using the abrasive water injection jet. The material testing was made using a universal tension/ pressure testing machine with a $250 \mathrm{kN}$ load cell. The testing speed was $2 \mathrm{~mm} / \mathrm{min}$. 


\section{FEASIBILITY STUDY RESULTS}

\subsection{Dry Ice Blasting Results}

The experimental findings of the dry ice blasting tests showed that the method offers enough power for the layer removal of CFRP. During the stationary operation, a complete removal of all layers was reached even at low pressures $(0.2$ to $0.5 \mathrm{MPa})$. Blasting pressures of more than 1.1 MPa resulted in a large-area material damage, caused by the high kinetic energy of the dry ice particles. The bottom layer was detached from the laminate and started to vibrate. This caused an uncontrolled delamination. The best results were reached either with a high feed rate and medium pressure $(0.6$ to $1 \mathrm{MPa})$, or with a low feed rate and low pressure $(0.2$ to $0.5 \mathrm{MPa})$. Both variants required multiple cycles.

The edge sharpness of the blasting results could be improved significantly by using a metal mask in the process. Although the blasting results with a fiber-corresponding feed direction were slightly more continuous than in the orthogonal direction, none of the tested parameter sets could provide a homogenous and defined material removal. Position and depth of the abrasion track were subject to fluctuations, which partially included multiple layers (Fig. 4). For this reason, no further experiments were performed using this method.

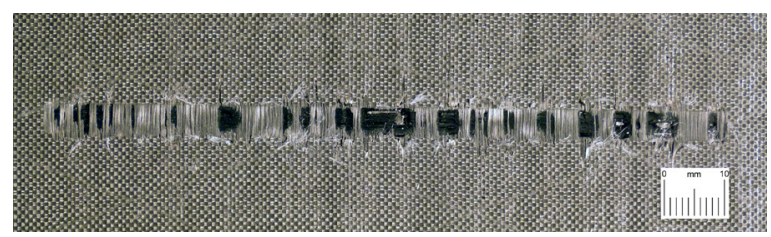

Fig. 4. Exemplary abrasion track after dry ice blasting of CFRP

\subsection{Snow Blasting Results}

By the first experiments it could be shown that the snow blasting method met its performance limits at the abrasion of CFRP. At low blasting pressures $(0.2$ to $0.5 \mathrm{MPa}$ ) no appreciable erosion was recognized with any parameter combination. Medium blasting pressures $(0.6$ to $\mathrm{MPa})$ in combination with low working distances ( 30 to $40 \mathrm{~mm}$ ) led to a slow removal of the first CFRP layer in stationary operation. The second layer could be laid open after a blasting time of 4 minutes. Unfortunately, even the slowest feed rate of $0.25 \mathrm{~mm} / \mathrm{s}$ was not sufficient for a satisfactory material removal during moving operations. The removal of the first layer in movement could only be reached at high blasting pressures (1.1 to $1.5 \mathrm{MPa}$ ) combined with a low feed rate of $0.25 \mathrm{~mm} / \mathrm{s}$. The best results using this method could be achieved with the $6 \mathrm{~mm}$ round nozzle, which offers the highest power density. An example is pictured in Fig. 5.

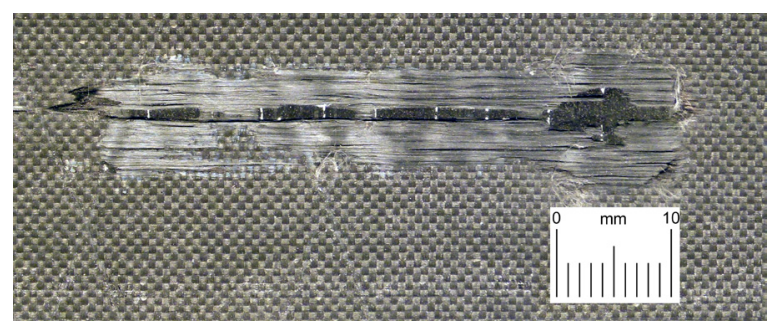

Fig. 5. Exemplary abrasion track after snow blasting of CFRP

Basically, it was determined that the tested parameter sets do not permit a constant and geometrically controlled material removal. Especially, the reproducibility was not given in repeated experiments. This can be attributed to the discontinuity of the blast, which is influenced by the breaking down of icings from the nozzle. The geometric position and depth of the erosion could not be controlled.

\subsection{Water Jet Results}

With regard to the snow- and dry ice- blasting processes, the water jet offers a significantly higher erosive potential. Pressures of more than $100 \mathrm{MPa}$ in combination with the tested standard sapphire nozzles effected an immediate cut-through. After the pressure range was reduced to 30 to $45 \mathrm{MPa}$, it was possible to remove single material layers with high feed rates.

The combination of the water jet's small processing area together with the exact positioning of the guiding machine allows a precise control of the material removal. Also according to the constancy this method shows a comparatively continuous jet form.

In the experiments it was found that the quality of the produced surfaces depends on multiple factors. A notable finding was that the tool manipulation in fiber direction $\left(0^{\circ}\right)$ resulted in more constant abrasion tracks than with orthogonal manipulation $\left(90^{\circ}\right)$. It was possible to lay open both, the second and the third material layer in one working step. Additionally, the surface quality was investigated in dependence of a track offset $\Delta x$ and pressure $p$. For the $0.25 \mathrm{~mm}$ round nozzle, which offers a significantly higher performance than the $0.1 \mathrm{~mm}$ nozzle, the optimum was found to be $\Delta x=0.3 \mathrm{~mm}$ (Fig. 6).

The nozzle standoff distance was $60 \mathrm{~mm}$. An offset that is too big results in a complete removal of 
the first layer and a severe structuring of the second one. A pressure reduction in combination with $0^{\circ}$ manipulation and the use of a $0.1 \mathrm{~mm}$ nozzle resulted in a micro-structuring of the first layer. This allowed detaching entire fiber bundles from the second layer.

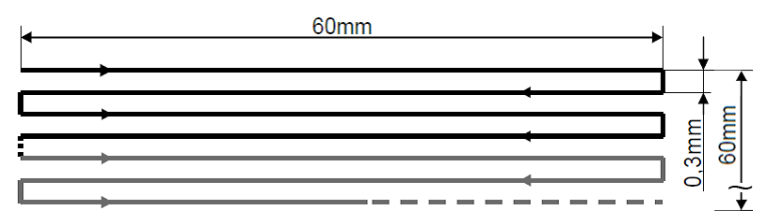

Fig. 6. Tool path for the surface preparation of an area

\section{PARAMETER STUDY ANALYSES AND DISCUSSION}

Generally speaking, very constant surfaces could be produced using the water jet method. Based on these promising results, the surface preparation of CFRP with the water jet was subject of a parameter study in the following.

Hereby the influence of important process parameters could be investigated in view of the main criteria "abrasion depth" and "damaging". The target was to determine a parameter set, which permits an optimal layer removal as well as a minimal variation of the results. The considered parameters were:

- $\quad$ Standoff distance $z$;

- Water pressure $p$;

- Feed rate $f$.

Additionally, the influence of the manipulating direction was investigated $\left(0^{\circ}\right.$ respectively $90^{\circ}$ to the first fiber layer). The nozzle diameter of $0.25 \mathrm{~mm}$ remained constant. For the parameter studies, fivelayered CFRP laminates with a layer thickness of 0.3 $\mathrm{mm}$ and a $\left(0^{\circ} / 90^{\circ} / 0^{\circ} / 90^{\circ} / 0^{\circ}\right)$ layer orientation were used. These laminates were manufactured using the hot press method. Every material removal track was measured in five positions.

Regarding Fig. 7 it becomes apparent that the influence of the standoff distance is affected by the manipulating direction in a severe way. This can be reasoned with a selective layer removal, which is favored at feed directions in fiber orientation. In this case, the first layer (thickness: $300 \mu \mathrm{m}$ ) is steadily removed, while the second fiber layer is not damaged by the decreased energy of the water jet. In case of an orthogonal feed rate direction in reference to the upper fiber orientation, the second layer is in favored position. Consequently, the remaining energy is still sufficient to cause an appreciable material removal depth of almost $150 \mu \mathrm{m}$ to this layer.

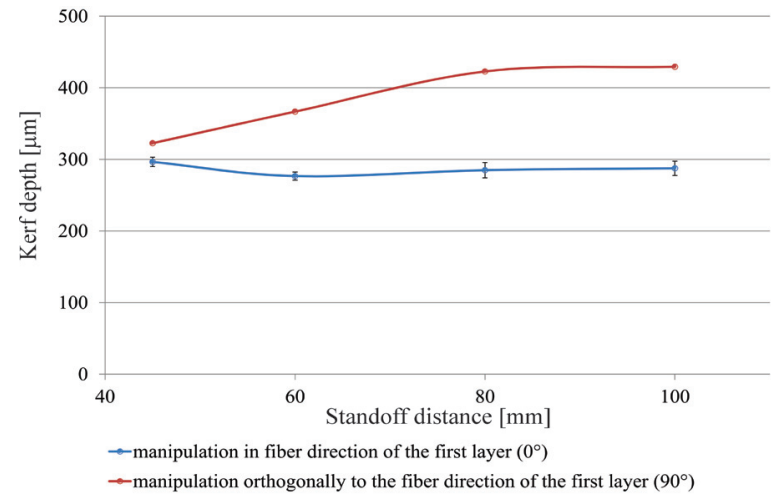

Fig. 7. Kerf depth in dependence of the standoff distance (pressure: $45 \mathrm{MPa}$, feed rate: $11.67 \mathrm{~mm} / \mathrm{s}$ )

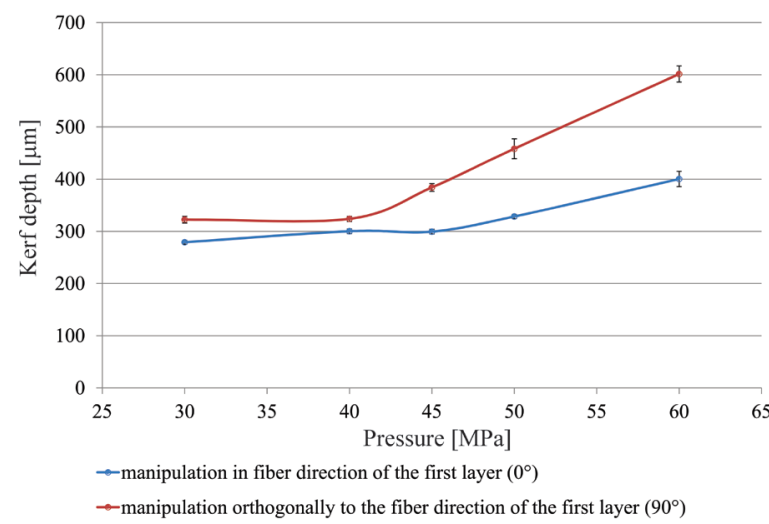

Fig. 8. Kerf depth in dependence of the water pressure (standoff: $60 \mathrm{~mm}$, feed rate: $11.67 \mathrm{~mm} / \mathrm{s}$ )

As pictured in Fig. 8, a rise of the kerf depth can be observed with increasing water pressure. This fact confirmed the expectations based on the dependence between jet velocity and pressure. Furthermore, the test results confirm the assumptions of the selective layer removal made in the last paragraph.

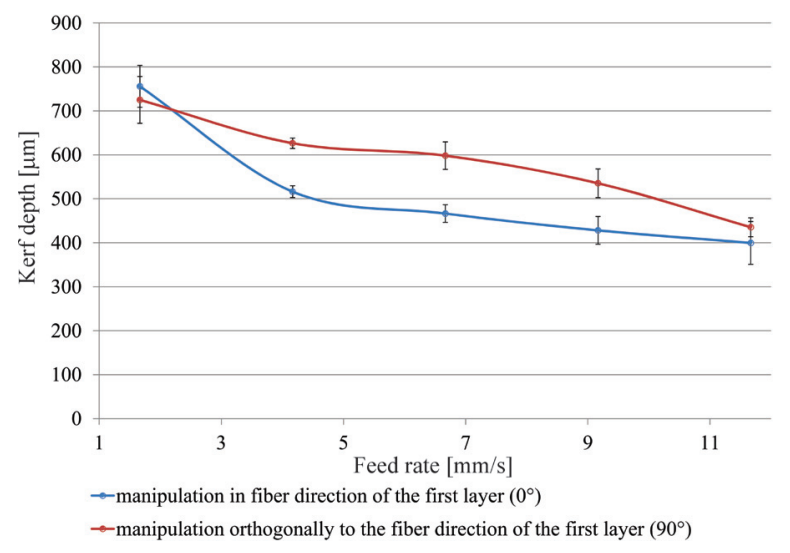

Fig. 9. Kerf depth in dependence of the feed rate (stand off distance: $60 \mathrm{~mm}$, pressure: $45 \mathrm{MPa}$ ) 
The third main factor in the parameter study was the feed rate, which was varied in five steps. The feed rate of $11.67 \mathrm{~mm} / \mathrm{s}$ presented the maximum value specific for the guiding machine used. Founded on the assumption that the contact time defines the material removal rate at a constant material removal potency, a decreasing curve progression was expected for increasing feed rates (Fig. 9).

In reference to the other parameter studies in respect of standoff distance and water pressure, the measured kerf depths in Fig. 9 are slightly higher. This can be explained by the way the parameter tests were arranged. Since the low feed rates were tested at first and the following experiment's starting point matched the former end point, the erosion was initiated in a more damaged location at the beginning. Through this approach a higher material removal rate by usage of the same jetting parameters could be achieved.

Finally, the parameter set for the removal of single material layers should be chosen according to the following two criteria:

1. the complete layer should be removed, and

2. the subjacent layers should not be damaged.

Based on these criteria, a parameter set was chosen and this allowed certain tolerances for pressure fluctuations and standoff distance variations. The test specimens were prepared for repair using this parameter set.

\subsection{Repair of CFRP Laminates}

Subsequent to the successful machining of the stepped peripheral zone, first repair experiments were made. Two $\left(0^{\circ} / 90^{\circ} / 90^{\circ} / 0^{\circ}\right)$ 4-layer CFRP laminates with a layer thickness of 0.3 $\mathrm{mm}$ were prepared using the above method. The step widths were $10 \mathrm{~mm}$ (specimen 1) respectively $20 \mathrm{~mm}$ (specimen 2). Afterwards, they were repaired with fitted prepreg patches. The laminates were hardened using the hot press method with a platform load of $26.1 \mathrm{kN}$. A preferable homogenous load distribution was provided by a polysiloxane mat between laminate and press mould. At the beginning, the platforms were heated to $60{ }^{\circ} \mathrm{C}$ (base temperature). From this point, the laminates were heated up to the hardening temperature of $110{ }^{\circ} \mathrm{C}$ and later cooled down to 60 ${ }^{\circ} \mathrm{C}$ again. The hardening temperature was held for 60 minutes, while heating-up and cooling down required 30 minutes each. Along with the mentioned laminates, a reference specimen was manufactured to symbolize an idealized repair method. The stepped peripheral zone was cut manually into each prepreg layer of this reference laminate before the hardening process.
Also, before the hardening, the prepreg repair patches were inserted. Base laminate and repair patches were hardened simultaneously. Tensile tests were made with the test material, the reference material and an undamaged CFRP laminate.

\section{CONCLUSIONS}

Regarding the parameter test results, the manipulation direction has a bigger influence on the material removal than e.g. the nozzle standoff distance. If possible, this should be utilized. The parameter set should allow a way of tolerance for pressure variations.

With the chosen parameters, the selective removal of single layers and the manufacturing of a stepped peripheral zone were possible (Fig. 10).

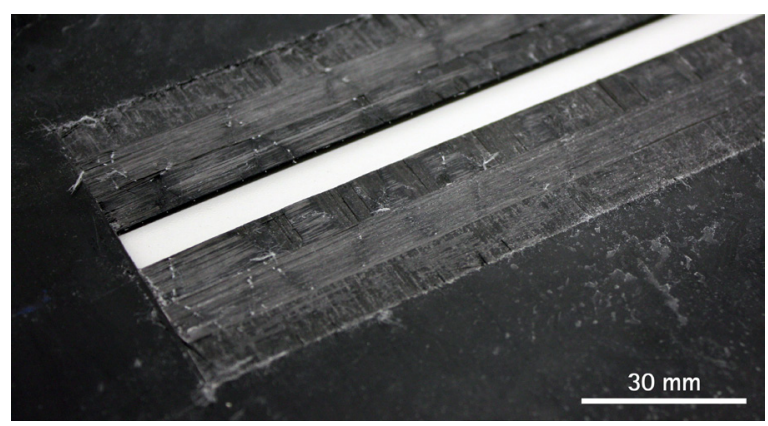

Fig. 10. Stepped peripheral zone (CFRP) after machining with the pure water jet

While the repaired reference laminates with the $10 \mathrm{~mm}$-stepping had a residual tensile strength of $24 \%$ (244 MPa) in comparison to the undamaged material, the water jet machined specimen still had a residual strength of $20 \%$ (197 MPa). The values for the $20 \mathrm{~mm}$ stepping were a little higher, the strength amounted 29\% (294 MPa, reference laminate) respectively $26 \%$ (263 MPa, water jet machined laminate). The undamaged CFRP laminate had a measured tensile strength of $1004 \mathrm{MPa}$.

When comparing the mechanical values of the repaired laminates (idealized and water jet prepared) it becomes apparent that the water jet method allows an average of $86 \%$ remaining tensile strength compared to the idealized laminate. Through this, it can be shown that the water jet machining offers a lot of potential for the surface preparation of CFRP laminates. Compared to an undamaged laminate, the remaining tensile strength is significantly lower. Certainly, this point can also be applied to the repaired laminates using the idealized method and is therefore not machining-dependent. It should also be considered 
that the repaired areas have not been supplied by external repair plies as has been done by other authors [2]. Since the geometrical properties of the repaired laminate should correspond with the original values, a solution without these external plies is preferable. The selective layer removal with the pure water jet allows the machining of many different geometries and designs for the transition zone. This offers a lot of research potential for the future.

\section{ACKNOWLEDGEMENT}

The authors are members of the Waterjet Laboratory Hannover (WLH) and of the German Working Group of Waterjet Technology (AWT).

The results presented here were acquired within a research project financed by the German Research Foundation (DFG). The authors would like to thank the DFG for their support.

\section{REFERENCES}

[1] Ehrenstein, G.W. (2006). Faserverbund-Kunststoffe. Werkstoffe - Verarbeitung - Eigenschaften. 2. Auflage. Carl Hanser Verlag, München, Wien.

[2] Ahn, S.-H., Springer, G.-S. (1998). Repair of Composite Laminates. Journal of Composite Materials, vol. 32, p. 1035-1114.

[3] Ahn, S.H., Springer, G.S., Shyprykevic, P. (1996). Composite Repair with Wet Lay-Up and Prepreg.
Composites '96 Manufacturing and Tooling Conference, Society of Manufacturing Engineers, p. 211-226.

[4] Baker, A. (2006). Development of a Hard-Patch Approach for Scarf Repair of Composite Structure. Defence Science and Technology Organisation, Canberra.

[5] Wang, C.-H., Gunnion, A.-J. (2008). On the design methodology of scarf repairs to composite laminates. Composites Science and Technology, vol. 68, p. 35-46, DOI:10.1016/j.compscitech.2007.05.045.

[6] Öjmertz, C. (1997). A Study on Abrasive Waterjet Milling. Doctoral Thesis. Chalmers University of Technology, Gothenburg.

[7] Borkowski, P. (2010). Novel Technique for Spatial Objects Shaping With High-Pressure Abrasive Water Jet. Strojniški vestnik - Journal of Mechanical Engineering, vol. 56, no. 5, p. 287-294.

[8] Momber, A.W., Schulz, R.-R. (2006). Handbuch der Oberflächenbearbeitung Beton. Birkhäuser Verlag, Basel.

[9] Ligocki, A. (2005). Schneiden landwirtschaftlicher Güter mit dem Hochdruckwasserstrahl. Shaker Verlag, Aachen.

[10] Tönshoff, K., Kroos, F., Marzenell, C. (1997). Highpressure Water Peening - a New Mechanical SurfaceStrengthening Process. CIRP Annals - Manufacturing Technology, vol. 46, no. 1, p. 113-116.

[11] Momber, A.W., Kovacevic, R. (1998). Principles of Abrasive Water Jet Machining. Springer-Verlag Limited, London, DOI:10.1007/978-1-4471-1572-4. 